\title{
Repetitive Evaluation of Fibrillation Cycle Length Predicts the Effi- cacy of Bepridil for Interruption of Long-Lasting Persistent Atrial Fibrillation
}

\author{
Yuya Aoyama, ${ }^{1}$ MD, Shinichi Niwano, ${ }^{1}$ MD, Hiroe Niwano, ${ }^{1}$ MD, Akira SATOH, ${ }^{1}$ MD, \\ Jun Kishihara, ${ }^{1}$ MD, Shoko Ishikawa, ${ }^{1}$ MD, Masami Murakami, ${ }^{1}$ MD, Kyoko Fukumoto, ${ }^{2}$ PhD, \\ Kazuyuki Ueno, ${ }^{2} \mathrm{PhD}$, and Tohru IzumI, ${ }^{1} \mathrm{MD}$
}

\begin{abstract}
SUMMARY
Although bepridil is effective for conversion of long-lasting persistent atrial fibrillation (AF) to sinus rhythm, it sometimes takes a long time to interrupt $\mathrm{AF}$ and there is no feasible index to predict its efficacy.

In 60 patients with long-lasting persistent $\mathrm{AF}$, bepridil (100-200 mg/day) was administered and continued for 8 weeks while body surface ECG was recorded every 2 weeks. The fibrillation cycle length (FCL) was evaluated using the spectral analysis of the fibrillation waves in each ECG. AF was interrupted in 32 patients receiving bepridil. The conversion was observed at 2 weeks in 4 , at 4 weeks in 7 , at 6 weeks in 7 , and at 8 weeks in 14 patients. When comparing these responders and nonresponders, clinical background characteristics other than the dosage of bepridil did not show any difference and neither did temporal changes in QT parameters and heart rate. In contrast, the FCL and $\triangle$ FCL (prolongation in FCL from baseline) became significantly larger in responders than in nonresponders at later observation points (FCL: $177 \pm 17$ versus $164 \pm 19 \mathrm{~ms}, P=0.018$, and $\Delta \mathrm{FCL}: 38 \pm 16$ versus $22 \pm 12 \mathrm{~ms}, P<0.001$, at 4 -week point; FCL: $188 \pm 17$ versus $169 \pm 19 \mathrm{~ms}, P=0.004$, and $\Delta \mathrm{FCL}: 49 \pm 18$ versus $27 \pm 14 \mathrm{~ms}, P<0.001$, at 6 -week point).

Repetitive evaluation of FCL using spectral analysis of fibrillation waves can be a feasible index to predict the efficacy of bepridil therapy. (Int Heart J 2011; 52: 353-358)
\end{abstract}

Key words: Persistent atrial fibrillation, Bepridil, Fibrillation cycle length, Spectral analysis

$\Lambda$ lthough bepridil was first introduced as an antianginal agent with a calcium channel blocking effect, ${ }^{1,2}$ recent studies have demonstrated that it might be effective for interrupting long-lasting persistent atrial fibrillation $(\mathrm{AF}){ }^{3,4)}$ This antiarrhythmic action is considered to appear through the blocking effects of several types of potassium and sodium channels $\mathrm{s}^{5-7)}$ and these effects would result in prolongation of the action potential duration and the refractory period in the atria and ventricles. ${ }^{8}$ In addition to these electrophysiological actions, it has been speculated in a few reports that bepridil might affect the myocardial remodeling that would promote the arrhythmogenic substrates in a condition of long-lasting $\mathrm{AF}$, and the recovery of down-regulated ion channels was induced by bepridil treatment at least in several experimental studies. ${ }^{9,10)}$ Regardless of the validity of the proposed anti-remodeling effect of bepridil, several reports have confirmed that it sometimes takes a relatively long time for bepridil to interrupt long-lasting AF even in effective cases. Additionally, bepridil therapy could be associated with risk of adverse effects such as torsades de pointes, QT prolongation, or bradycardia. ${ }^{11)}$ Therefore, the prediction of bepridil efficacy is important to avoid unnecessary use of bepridil in resistant cases. We previously reported the importance of the fibrillation cycle length
(FCL) evaluated using spectral analysis of the fibrillation waves in surface ECG as an index of the atrial electrophysiological properties, and tried to predict the efficacies of antiarrhythmic agents, comparing bepridil and pilsicainide in cases with persistent $\mathrm{AF}^{12)} \mathrm{As}$ a result, it was shown that FCL could be a useful index to predict the efficacy of pilsicainide in 4-week observation, but was not sufficient for bepridil therapy, most likely because of the relatively short-term and limited observation. In the present study, the FCL during bepridil therapy was repeatedly evaluated in a prolonged observation period, and the usefulness of repetitive FCL measurement as a predictor of bepridil efficacy was evaluated in a prospective manner.

\section{Methods}

Study population: The study population consisted of 60 consecutive patients with AF lasting longer than 2 months. The clinical characteristics of the total population are summarized in Table I. Fifteen of the 60 patients were female, and the mean age was $65 \pm 9$ years. The duration of $\mathrm{AF}$ was estimated from the history of clinical symptoms and ECG recordings, and the mean duration was $8.1 \pm 5.8$ months. Underlying heart disease

From the ${ }^{1}$ Department of Cardio-angiology, Kitasato University School of Medicine, Kanagawa and ${ }^{2}$ Department of Pharmaceutical Sciences, Niigata University of Pharmacy and Applied Life Sciences, Niigata, Japan.

Address for correspondence: Yuya Aoyama, MD, Department of Cardio-angiology, Kitasato University School of Medicine, 1-15-1 Kitasato, Minami-ku, Sagamihara, Kanagawa 252-0374, Japan.

Received for publication August 18, 2011.

Revised and accepted September 22, 2011 
was observed in 29/60 patients, namely, hypertension in 29, and ischemic heart disease in 3 patients. In 16/60 patients, 1-2 types of Class Ia or Ic antiarrhythmic agents, namely, pilsicainide in 9 patients, flecainide in 3, propafenone in 1, disopyramide in 3 , and cibenzoline in 3 , were prescribed for the purpose of recovery of sinus rhythm and failed to interrupt AF. In the remaining 44 patients, bepridil was used as the initial rhythm control medicine. Before enrollment in the present study, all patients underwent routine examinations including transthoracic echocardiography and the patients who met any of the following criteria were excluded: 1 ) age $>80$ years; 2) patients with $\mathrm{AF}$ persisting $>3$ years; 3) patients with left ventricular dysfunction $(\mathrm{LVEF}<40 \%)$ or history of congestive heart failure; 4) patients with QT interval prolongation $(>450 \mathrm{~ms})$ on baseline ECG; 5) patients with bradycardia ( $<50$ beats/minute) or severe conduction system disturbance; 6) patients with severe valvular heart disease; 7) patients with thrombus formation in the heart; and 8) patients with severe hepatic or renal dysfunction. All studies were performed with the approval of the Clinical Studies and Ethics Committee of Kitasato University Hospital and written informed consent was obtained at the time of enrollment in the study.

Study protocol: All antiarrhythmic agents were discontinued for more than 5 times the half-lives of each medicine before the start of the study protocol. Concomitant use of digitalis, a $\beta$ -blocker, or a calcium blocker was allowed as rate control therapy if required. Under the continuation of optimal anticoagulation therapy for more than 3 weeks, oral bepridil at 100-200 $\mathrm{mg} /$ day was started and continued for 8 weeks. The dose of bepridil was determined by a physician, taking into considera-

Table I. Clinical Characteristics of the Patients

\begin{tabular}{|c|c|c|c|c|}
\hline & $\begin{array}{c}\text { Total } \\
(n=60)\end{array}$ & $\begin{array}{c}\text { Respond- } \\
\text { ers } \\
(n=32)\end{array}$ & $\begin{array}{c}\text { Nonre- } \\
\text { sponders } \\
(n=28)\end{array}$ & $P$ \\
\hline Age (years) & $65 \pm 9$ & $65 \pm 8$ & $65 \pm 11$ & 0.926 \\
\hline Gender (F/M) & $15 / 45$ & $7 / 25$ & $8 / 20$ & 0.550 \\
\hline Duration of AF (months) & $8.1 \pm 5.8$ & $7.2 \pm 4.9$ & $9.2 \pm 6.0$ & 0.152 \\
\hline \multicolumn{4}{|l|}{ Underlying disease } & $0.011^{\prime}$ \\
\hline Hypertension, $n(\%)$ & $29(48)$ & $17(53)$ & $12(43)$ & 0.427 \\
\hline $\mathrm{IHD}, n$ & 3( & & & 0.475 \\
\hline None, $r$ & $31(52)$ & $15(47)$ & $16(57)$ & 0.427 \\
\hline CHADS2 score & $0.8 \pm 0.8$ & $0.8 \pm 0.8$ & $0.9 \pm 0.9$ & 0.420 \\
\hline \multicolumn{5}{|l|}{ ECG parameters } \\
\hline HR (bpm) & $86 \pm 13$ & $87 \pm 13$ & $86 \pm 13$ & 0.813 \\
\hline QT interval (ms) & $345 \pm 25$ & $344 \pm 25$ & $348 \pm 25$ & 0.557 \\
\hline $\mathrm{QTc}\left(\mathrm{ms} / \mathrm{s}^{1 / 2}\right)$ & $414 \pm 22$ & $411 \pm 23$ & $417 \pm 22$ & 0.273 \\
\hline FCL (ms) & $142 \pm 13$ & $141 \pm 13$ & $142 \pm 13$ & 0.742 \\
\hline \multicolumn{5}{|l|}{$\underline{T T E}$ parameters } \\
\hline $\mathrm{LAD}(\mathrm{mm})$ & $45.2 \pm 6.3$ & $44.4 \pm 7.3$ & $46.1 \pm 4.9$ & 0.327 \\
\hline $\operatorname{LVEF}(\%)$ & $65.5 \pm 5.5$ & $65.6 \pm 5.6$ & $65.3 \pm 5.5$ & 0.848 \\
\hline $\mathrm{BNP}(\mathrm{pg} / \mathrm{mL})$ & $114 \pm 57$ & $113 \pm 53$ & $115 \pm 62$ & 0.915 \\
\hline \multicolumn{5}{|l|}{ Prescribed medicines } \\
\hline ACEIs/ARB & 4. & $14(44)$ & $10(36)$ & 0.526 \\
\hline$\beta$-Blocke & 19 & & $12(43)$ & 0.081 \\
\hline Ca-blocker, $n(\%)$ & $14(23)$ & $6(19)$ & $8(29)$ & 0.370 \\
\hline
\end{tabular}

All data shown were evaluated before bepridil administration. IHD indicates ischemic heart disease; LAD, left atrial dimension; LVEF, left ventricular ejection fraction; BNP, brain natriuretic peptide; ACEI, angiotensin converting enzyme inhibitor; and ARB, angiotensin receptor blocker. Asterisk indicates statistical significance. tion age, gender, body weight, renal function, and other factors. Bepridil was basically administered in an attempt to convert $\mathrm{AF}$ to sinus rhythm, but was continued for 8 weeks even after AF interruption was achieved to maintain the sinus rhythm unless obvious adverse complications including QT prolongation $(>500 \mathrm{~ms}$ ), bradycardia ( $<50$ beats/minute), or torsades de pointes appeared. During the administration of bepridil, the 12-lead ECG recording was repeated every 2 weeks for evaluation of heart rhythm and ECG parameters including heart rate and QT interval. The serum level of potassium was also measured to avoid low-potassium-induced QT prolongation or torsades de pointes. When AF was continued at the time of ECG recording, the specific ECG recording as described below was performed for spectral analysis of the fibrillation waves. In accordance with the methodology of this study, the FCL could be evaluated only in patients with AF rhythm. Although the QT interval and the heart rate could be evaluated regardless of the heart rhythm, the QT interval and the heart rate were evaluated only in patients with AF rhythm to evaluate the electrophysiological parameters in the same population as the FCL evaluation. When AF interruption and conversion to sinus rhythm were achieved within the 8-week protocol, such patients were classified as responders, and the patients without sinus rhythm recovery were classified as nonresponders.

Spectral analysis of the fibrillation waves: In patients who exhibited AF rhythm in the standard 12-lead ECG, an additional 41-second recording of the V1 lead was performed for spectral analysis of the fibrillation waves. Data were digitally transmitted through an A/D exchanger for storage in a computer system at a sampling rate of $1 \mathrm{kHz}$. The QRS-T complex was subtracted using a template matching algorithm ${ }^{12,13)}$ to extract the pure fibrillation wave for the analysis. Frequency analysis was performed off-line on a microcomputer (Bimutas II, Kissei Co. Ltd., Matsumoto, Japan) as previously described. ${ }^{12)}$ The frequency analysis of the subtracted ECG involved 3 steps: (1) band-pass filtering, (2) application of a Hamming window, and (3) 4096-point fast Fourier transformation. A 50\% overlap of adjacent spectral analyses allowed the use of an average of 20 epochs of analyses within a single 44-second data set. After spectral analysis, the power spectra were quantified by measuring the peak frequency signal with the maximum magnitude derived from each epoch. The peak frequency of the spectrum in the $3-12 \mathrm{~Hz}$ range was converted to a cycle length (CL in ms $=1,000 /$ frequency), defined as the fibrillation CL (FCL), and was averaged from 20 epochs. As the index for the change in FCL during the follow-up period, $\triangle$ FCL was calculated by subtracting the baseline FCL from the FCL at each evaluation time point.

Statistical analysis: All values are expressed as the mean \pm standard deviation. Statistical analysis was performed with the 1 -way ANOVA test. A $P$ value of $<0.05$ was considered statistically significant.

\section{RESUlts}

Efficacy of bepridil for pharmacological cardioversion: Of the 60 patients, 22 were assigned to $100 \mathrm{mg} /$ day bepridil, and the remaining 38 were assigned to $200 \mathrm{mg} /$ day (mean dosage: 163 $\pm 48 \mathrm{mg}$ /day). $\beta$-Blockers, calcium blockers, and angiotensin converting enzyme inhibitors (ACEI)/angiotensin II receptor 


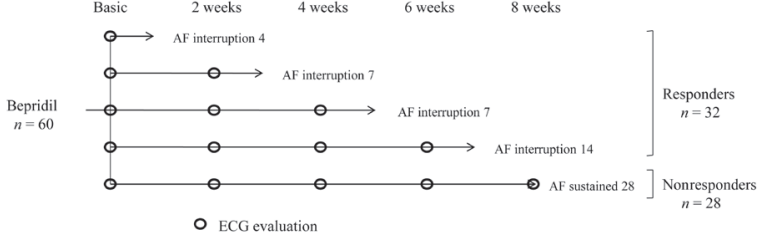

Figure 1. Schematic time course of 60 patients who underwent bepridil therapy. AF interruption was observed at the 2-week time point in 4 patients, 4 weeks in 7, 6 weeks in 7, and 8 weeks in 14 patients, and AF was not interrupted in the remaining 28 patients. In this study, all patients with $\mathrm{AF}$ interruption were classified as responders and those without AF interruption as nonresponders. See text for details. AF indicates atrial fibrillation and ECG, electrocardiogram.
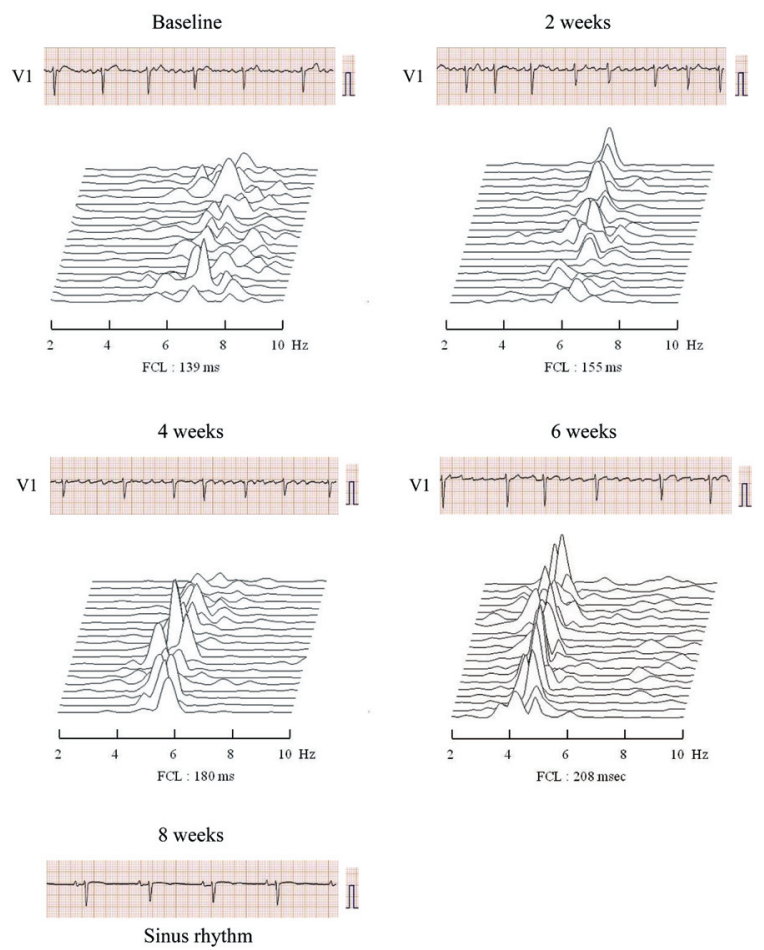

Figure 2. Surface ECGs and results of spectral analysis of the fibrillation waves in a representative case. At the baseline analysis, the fibrillation wave was fine and the FCL was 139 ms. During bepridil therapy, the configuration of the fibrillation gradually became coarse and the FCL was also prolonged gradually. Finally, at the 6-week time point, the FCL was $208 \mathrm{~ms}$, and then AF was interrupted at 8 weeks. See text for discussion. FCL indicates fibrillation cycle length and AF, atrial fibrillation.

blockers (ARB) were prescribed in 19, 14, and 24 patients, respectively, for the purpose of rate control or treatment of hypertension (Table I). The conversion of AF to sinus rhythm was achieved in $32 / 60$ patients $(53 \%)$ within the 8 -week proto$\mathrm{col}$. The time point of conversion to sinus rhythm was 2 weeks in 4 patients, 4 weeks in 7, 6 weeks in 7, and 8 weeks in the remaining 14 patients (Figure 1). As a result, 32 patients with $\mathrm{AF}$ interruption were classified as responders, and the remaining 28 patients without AF interruption were classified as nonresponders. Because the FCL could be evaluated only during AF

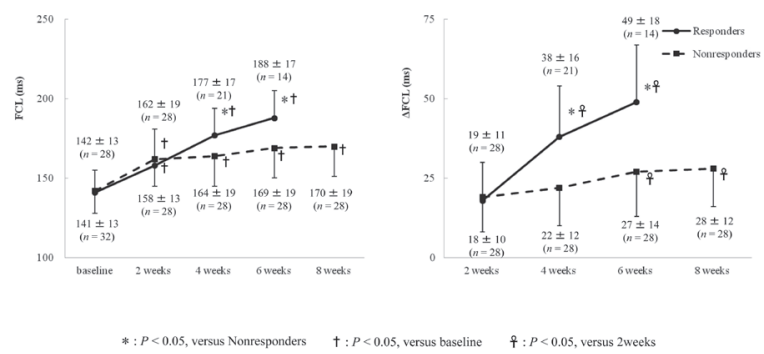

Figure 3. Changes in FCL-related data in responders and nonresponders along the time course of bepridil therapy. The FCL did not differ between the two groups at the baseline and 2-week observation points, but the FCL in the responders became significantly longer than that in the nonresponders at later observation points. There was no significant difference in $\Delta$ FCL between the two groups at the 2-week observation point, although it did become larger in the responders than in the nonresponders at later observation points. The FCL had become larger than that at the baseline after administration of bepridil in both groups. The $\triangle \mathrm{FCL}$ at later observation points had become larger than that at the 2 -week observation point. See text for details. FCL indicates fibrillation cycle length.
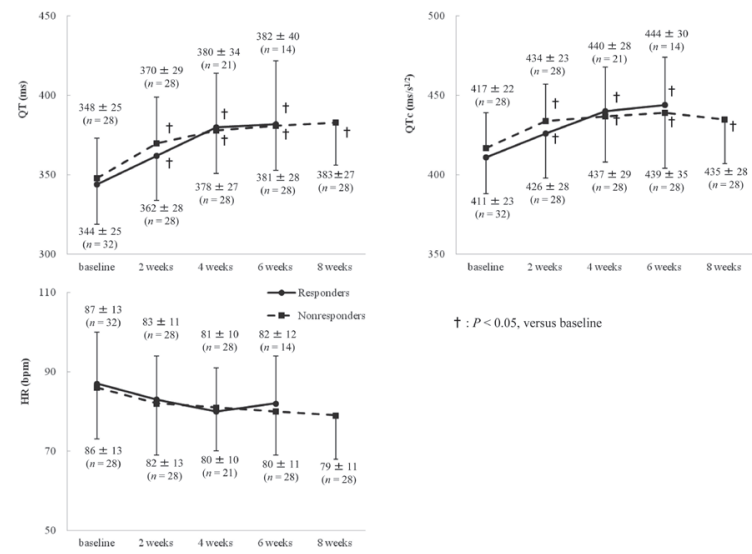

Figure 4. Changes in QT, QTc, and HR in responders and nonresponders along the time course of bepridil therapy. There was no significant difference between the two groups at all observation points along the time course. The QT and QTc became longer than those at baseline after administration of bepridil in both groups. In contrast, the heart rate tended to show a gradual decrease along the time course. See text for details. QT indicates QT interval; QTc, corrected QT interval; and HR, heart rate.

rhythm, the numbers of patients in whom FCL could be evaluated were 28 at the 2-week time point, 21 at 4 weeks, 14 at 6 weeks, and 0 at 8 weeks in the responders. During the 8 -week protocol, no patient exhibited any adverse complications such as abnormal QT prolongation, negative change in T-U wave morphology, or torsades de pointes.

Clinical characteristics of the responders and nonresponders: The clinical characteristics of the responders and nonresponders for bepridil therapy are summarized in Table I. There was no significant difference between the two groups in the basic characteristics including age, gender, duration of AF, underlying disease, echo-cardiographic parameters, and concomitantly prescribed medicines, but the dose of bepridil was higher in responders than in nonresponders $(178 \pm 42$ versus $146 \pm 50 \mathrm{mg}$ / 


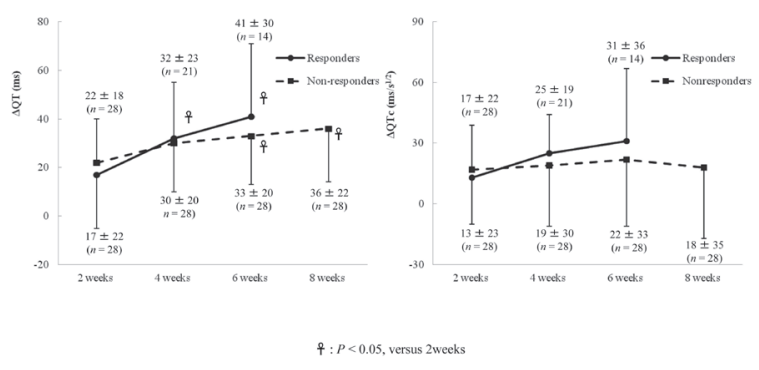

Figure 5. Changes in $\Delta \mathrm{QT}$ and $\Delta \mathrm{QTc}$ in responders and nonresponders along the time course of bepridil therapy. The $\Delta \mathrm{QT}$ had become larger than that at baseline after administration of bepridil in both groups. $\triangle \mathrm{QTc}$ also tended to show a gradual increase along the time course, but there was no significant difference in its temporal change in both groups. Neither $\Delta \mathrm{QT}$ nor $\Delta \mathrm{QTc}$ showed any difference between the two groups. See text for details. QT indicates QT interval and QTc, corrected QT interval.

Table II. Univariate and Multivariate Analyses of the Index for AF Interruption at 4-Week Observation Point

\begin{tabular}{|c|c|c|c|c|}
\hline & \multirow{2}{*}{$\begin{array}{c}\text { Univari- } \\
\text { ate } \\
P\end{array}$} & \multicolumn{3}{|c|}{ Multivariate } \\
\hline & & $P$ & $\begin{array}{c}\text { Odds } \\
\text { ratio }\end{array}$ & $95 \% \mathrm{CI}$ \\
\hline Age $(<65$ years $)$ & 0.243 & 0.800 & 1.349 & $-1.083-1.332$ \\
\hline Duration of $\mathrm{AF}$ ( $<6$ months) & 0.058 & 0.080 & 6.589 & $-0.001-2.240$ \\
\hline Dose of bepridil (200 mg/day) & $0.036^{*}$ & 0.153 & 5.946 & $-0.262-2.262$ \\
\hline Lone AF & 1.000 & 0.289 & 0.293 & $-1.923-0.425$ \\
\hline $\operatorname{LAD}(<45 \mathrm{~mm})$ & 0.292 & 0.709 & 1.465 & $-0.839-1.228$ \\
\hline $\mathrm{HR}_{4 \text { week }}(<80 \mathrm{bpm})$ & 0.187 & 0.199 & 3.897 & $-0.304-1.847$ \\
\hline $\mathrm{QT}_{4 \text { week }}(>380 \mathrm{~ms})$ & 0.864 & 0.895 & 1.219 & $-1.436-1.597$ \\
\hline $\mathrm{QTc}_{4 \text { week }}\left(>440 \mathrm{~ms} / \mathrm{s}^{1 / 2}\right)$ & 0.322 & 0.234 & 0.115 & $-3.218-0.505$ \\
\hline $\mathrm{FCL}_{4 \text { week }}(>170 \mathrm{~ms})$ & $0.017^{*}$ & 0.169 & 4.413 & $-0.280-1.926$ \\
\hline$\Delta \mathrm{QT}_{4 \text { week }}(>40 \mathrm{~ms})$ & 0.458 & 0.390 & 2.329 & $-0.510-1.487$ \\
\hline$\Delta \mathrm{QTc}_{\text {4week }}\left(>25 \mathrm{~ms} / \mathrm{s}^{1 / 2}\right)$ & 0.680 & 0.557 & 2.198 & $-0.837-1.876$ \\
\hline$\Delta \mathrm{FCL}_{4 \text { week }}(>30 \mathrm{~ms})$ & $<0.001^{*}$ & $0.044^{*}$ & 8.951 & $0.103-2.330$ \\
\hline
\end{tabular}

$\mathrm{AF}$ indicates atrial fibrillation; $\mathrm{LAD}$, left atrial dimension; HR, heart rate; FCL, fibrillation cycle length; and 95\%CI, 95\% confidence interval. Asterisk indicates statistical significance.

day, $P=0.011)$.

FCL and ECG parameters in the responders and nonresponders: The baseline data of the FCL and ECG parameters, that is, heart rate and QT intervals, are exhibited in Table I; there was no difference in these parameters. Figure 2 shows the ECGs and results of spectral analysis of the fibrillation waves in a representative case in whom the AF was interrupted after 8 weeks of bepridil treatment. Figure 3 shows the FCL and $\Delta$ FCL data in responders and nonresponders along the time course. The FCL showed gradual prolongation along the time course and had become larger than that at baseline after administration of bepridil in both groups. The FCL data did not show a significant difference between the 2 groups at the observation points of baseline and 2 weeks, but the FCL in the responders became significantly longer than that in the nonresponders at later observation points ( 4 weeks: $177 \pm 17$ versus $164 \pm 19$ ms, $P=0.018$; 6 weeks: $188 \pm 17$ versus $169 \pm 19 \mathrm{~ms}, P=$ 0.004). The $\triangle \mathrm{FCL}$, that is, the prolongation in FCL during the bepridil therapy, did not show a significant difference between the 2 groups at the 2 -week observation point, but it became
Table III. Univariate and Multivariate Analyses of the Index for AF Interruption at 6-Week Observation Point

\begin{tabular}{|c|c|c|c|c|}
\hline & \multirow{2}{*}{$\begin{array}{c}\text { Univari- } \\
\text { ate } \\
P\end{array}$} & \multicolumn{3}{|c|}{ Multivariate } \\
\hline & & $P$ & $\begin{array}{c}\text { Odds } \\
\text { ratio }\end{array}$ & $95 \% \mathrm{CI}$ \\
\hline Age & 185 & 0.178 & 9.742 & $-0.434-3.039$ \\
\hline Duration of $\mathrm{AF}$ ( $<6$ months) & .274 & .198 & 5.411 & $-0.332-2.404$ \\
\hline Dose of bepridil ( $200 \mathrm{mg} / \mathrm{day})$ & 0.124 & .820 & 1.525 & $-1.642-2.265$ \\
\hline Lone AF & 0.273 & .390 & 0.260 & $-2.660-0.708$ \\
\hline $\mathrm{LAD}(<45 \mathrm{~mm})$ & 0.562 & 0.551 & 0.393 & $-2.267-1.028$ \\
\hline $\mathrm{HR}_{\text {6week }}(<80 \mathrm{bpm})$ & 0.827 & 0.754 & 0.661 & $-1.670-0.973$ \\
\hline $\mathrm{QT}_{6 \text { week }}(>380 \mathrm{~ms})$ & 0.657 & 0.551 & 0.269 & $-3.217-1.342$ \\
\hline $\mathrm{QTc}_{\text {6week }}\left(>440 \mathrm{~ms} / \mathrm{s}^{1 / 2}\right)$ & 1.000 & 0.127 & 0.014 & $-5.501-0.229$ \\
\hline $\mathrm{FCL}_{6 \text { week }}(>180 \mathrm{~ms})$ & $0.026^{*}$ & 0.339 & 3.687 & $-0.675-2.194$ \\
\hline$\Delta \mathrm{QT}_{\text {6week }}(>40 \mathrm{~ms})$ & 0.266 & 0.189 & 10.17 & $-0.436-3.255$ \\
\hline$\Delta \mathrm{QTc}_{\text {6week }}\left(>25 \mathrm{~ms} / \mathrm{s}^{1 / 2}\right)$ & 0.374 & 0.101 & 37.21 & $0.037-4.699$ \\
\hline$\Delta \mathrm{FCL}_{\text {6week }}(>40 \mathrm{~ms})$ & $<0.001^{*}$ & $0.047^{*}$ & 19.54 & $0.186-3.300$ \\
\hline
\end{tabular}

$\mathrm{AF}$ indicates atrial fibrillation; $\mathrm{LAD}$, left atrial dimension; $\mathrm{HR}$, heart rate; FCL, fibrillation cycle length; and 95\% CI, 95\% confidence interval. Asterisk indicates statistical significance.

larger in the responders than in the nonresponders at later observation points ( 4 weeks: $38 \pm 16$ versus $22 \pm 12 \mathrm{~ms}, P<$ 0.001 ; 6 weeks: $49 \pm 18$ versus $27 \pm 14 \mathrm{~ms}, P<0.001)$. In the responders, $\triangle \mathrm{FCL}$ had become significantly larger than that at 2 weeks at later observation points. In the nonresponders, the prolongation in FCL had become larger than that at 2 weeks since the 6-week observation point. Figure 4 shows the QT interval, QTc, and heart rate data in responders and nonresponders along the time course. The QT interval showed gradual prolongation along the time course and had become longer than that at baseline after administration of bepridil in both groups. Differently from the FCL and $\triangle \mathrm{FCL}$ data, there was no significant difference between the responders and the nonresponders at all observation points along the time course. The QTc interval data also exhibited a similar tendency to the QT interval data. Neither $\Delta \mathrm{QT}$ nor $\Delta \mathrm{QTc}$ showed any difference between the two groups (Figure 5). In contrast, the heart rate tended to show a gradual decrease along the time course in both groups, and no difference was observed between the two groups at all observation points along the time course. Tables II and III exhibit the results of univariate and multivariate analyses of the clinical parameters as indices for AF interruption at 4 and 6 weeks after administration of bepridil. At the 4-week observation point, univariate analysis showed that significant indexes for predicting $\mathrm{AF}$ interruption were a bepridil dosage of $200 \mathrm{mg} / \mathrm{day}, \mathrm{FCL}>170 \mathrm{~ms}$, and $\triangle \mathrm{FCL}>30 \mathrm{~ms}$. In the multivariate analysis, $\triangle \mathrm{FCL}>30 \mathrm{~ms}$ was the only significant index, indicating that this is an independent predicting factor for AF interruption. Similarly, at the 6-week observation point, univariate analysis indicated an FCL $>180 \mathrm{~ms}$ and $\triangle \mathrm{FCL}>40$ $\mathrm{ms}$ were significant predicting factors, while multivariate analysis showed an $\triangle \mathrm{FCL}>40 \mathrm{~ms}$ was the only independent predicting factor for $\mathrm{AF}$ interruption.

\section{Discussion}

Major findings: The present study evaluating FCL along the time course of bepridil therapy for long-lasting AF has docu- 
mented several unique findings. First, bepridil was effective for $\mathrm{AF}$ interruption in 32/60 (53\%) patients with long-lasting AF. Second, when comparing the responders and nonresponders, FCL did not differ at the earlier observation points but became different at later observation points despite gradual prolongation in both groups. Third, the degree of FCL prolongation $(\triangle \mathrm{FCL})$ was larger in responders than in nonresponders at later observation points. Finally, differences between the responders and nonresponders were not observed in terms of the other electrocardiographic parameters, such as QT, QTc, and HR.

Effect of bepridil on long-lasting AF: Several reports have documented that bepridil would be useful for conversion of AF to sinus rhythm even in patients with drug-resistant long-lasting $\mathrm{AF}^{3,4)}$ The efficacy of bepridil therapy has been reported to occur in a dose-dependent manner, and it was $38 \%$ and $69 \%$ with dosages of 100 and $200 \mathrm{mg} /$ day, respectively. ${ }^{14)}$ The mean dose of bepridil in the present study was $163 \pm 48 \mathrm{mg} / \mathrm{day}$, so the incidence of $\mathrm{AF}$ interruption seems to be compatible with the results of previous studies. ${ }^{14)}$

Mechanism of the effect of bepridil on atrial electrophysiological properties: The precise mechanism of the efficacy of bepridil on drug-resistant $\mathrm{AF}$ is unclear, but it should depend, at least in part, on the blocking effects of several types of ion channels because bepridil has been documented to be a multiion channel blocker. ${ }^{5-7)}$ In accordance with these reports, the sodium channel blocking effect of bepridil is weak ${ }^{15)}$ and the blocking effects on the L-type calcium channel and potassium channels, namely, Ikr, Iks, and Ito, are stronger, ${ }^{5,6,16)}$ so bepridil is considered to act as a class III or class IV antiarrhythmic agent rather than a class I antiarrhythmic agent. ${ }^{17)}$ By considering the reentrant mechanism in the atrium, the cycle length should strongly depend on the conduction velocity in the fixed reentrant circuit, and the sodium channel blocking action will directly prolong the cycle length through a decrease in the conduction velocity. ${ }^{18)}$ In contrast, the potassium channel blocking action will prolong the atrial refractoriness but not affect the conduction velocity, and it will not affect the cycle length either, at least in a fixed reentrant circuit. However, because the reentrant circuits in AF are randomly and functionally determined, prolonged refractoriness caused by the potassium channel blocking action will result in deletion of smaller circuits and enlargement of the main rotor of reentrant rhythm. ${ }^{19,20)}$ This change caused by the potassium channel blocking action will result in prolongation of the cycle length of the main reentrant circuit of AF by prolonging the spatial wave-length. ${ }^{21,22)}$ The FCL evaluated in the present study is not a direct index of actual cycle length of AF reentrant circuits, but a strong correlation between the FCL and atrial refractoriness and/or size of the reentrant circuit has been documented in previous studies. ${ }^{2324)}$ The present study and our previous report have documented marked prolongation in FCL by pilsicainide, and slight or gradual prolongation in FCL by bepridil. ${ }^{12)}$ These results seem to be compatible with the theories described above.

Prediction of responders and nonresponders: Previous reports have indicated that a relatively long period is sometimes required for bepridil to interrupt $\mathrm{AF}^{14)}$ and it took more than 6 weeks to interrupt AF in 21/32 responders in the present study. The mechanism of this slow appearance of the antiarrhythmic effect is unclear, but unnecessary use of bepridil, especially in nonresponders, should be avoided to prevent adverse side effects such as torsades de pointes. ${ }^{11)}$ Therefore, the distinction of responders and nonresponders, in other words, prediction of bepridil efficacy, is an important issue in clinical use. In the present study, the patient population was divided into responders and nonresponders based on the results of 8-week bepridil therapy and the clinical parameters were compared between them to determine the indices that could predict bepridil efficacy. The result was that the dose of bepridil was the only clinical parameter that exhibited a significant difference between the two groups in the baseline data. In our previous study, the FCL data at the baseline examination could distinguish the bepridil responders when pilsicainide responders had been eliminated from the patient population, ${ }^{12)}$ but this did not work in the comparison in the present study. However, the FCL data exhibited differences between the two groups at later observation points in this study. Fujiki, et al also indicated that an increase in FCL provided important information related to the termination of persistent AF by bepridil alone or in combination with aprindine. ${ }^{25)}$ They evaluated FCL before and after administration of bepridil alone or both bepridil and aprindine, and indicated that FCL was larger in drug effective cases than that in the others. Our present study has also demonstrated basically similar findings, but the difference in FCL between responders and nonresponders became significant during continuous and repeated observation of FCL. A decision concerning bepridil efficacy may be easier by referring to repetitive FCL evaluations along the time course of therapy, at least based on the data of the present study. These findings indicate that the changes in the atrial electrophysiological parameters induced by bepridil therapy might become an index with which to predict bepridil efficacy in the near future. In contrast, the QT, QTc, and HR data did not show any difference between the two groups at any observation point in this study. This indicates that the changes in the parameters of ventricular repolarization and/or AV nodal conduction did not differ between the two groups. Because the changes in the QT and QTc are considered to reflect the tissue concentration of bepridil, ${ }^{26)}$ the effect of bepridil on the atrial electrophysiological properties may depend on factors other than tissue concentration.

Limitations: This prospective study has a few limitations. First, because the FCL in our method could be calculated only under AF rhythm, the FCL and atrial electrophysiological parameters could not be evaluated in patients with AF interruption. Second, we did not examine spontaneous FCL changes in control patients without bepridil therapy, although it is reported to decrease gradually over time. Finally, the number of patients was limited and sampling bias could not be eliminated.

Conclusions: Bepridil, a multi-channel blocker, was effective for conversion of long-lasting AF to sinus rhythm in 53\% of patients, but it took more than 6 weeks in $66 \%$ of the responders. The repetitive evaluation of FCL through the FFT analysis of fibrillation waves in surface ECG was considered to be a feasible index with which to predict the efficacy of bepridil.

\section{REFERENCES}

1. Harder DR, Sperelakis N. Bepridil blockade of $\mathrm{Ca}^{2+}$-dependent action potentials in vascular muscle of dog coronary artery. J Cardiovasc Pharmacol 1981; 3: 906-14.

2. DiBianco R, Alpert J, Katz RJ, et al. Bepridil for chronic stable angina pectoris: results of a prospective multicenter, placebo-con- 
trolled, dose-ranging study in 77 patients. Am J Cardiol 1984; 53: 35-41.

3. Fujiki A, Tsuneda T, Sugao M, Mizumaki K, Inoue H. Usefulness and safety of bepridil in converting persistent atrial fibrillation to sinus rhythm. Am J Cardiol 2003; 92: 472-5.

4. Nakazato Y, Yasuda M, Sasaki A, et al. Conversion and maintenance of sinus rhythm by bepridil in patients with persistent atrial fibrillation. Circ J 2005; 69: 44-8.

5. Yatani A, Brown AM, Schwartz A. Bepridil block of cardiac calcium and sodium channels. J Pharmacol Exp Ther 1986; 237: 9-17.

6. Wang JC, Kiyosue T, Kiriyama K, Arita M. Bepridil differentially inhibits two delayed rectifier K (+) currents, I (kr) and I (ks), in guinea-pig ventricular myocytes. Br J Pharmacol 1999; 128: 1733 8 .

7. Kobayashi S, Reien Y, Ogura T, Saito T, Masuda Y, Nakaya H. Inhibitory effect of bepridil on hKv1.5 channel current: comparison with Amiodarone and E-4031. Eur J Pharmacol 2001; 430: 14957.

8. Kato R, Singh BN. Effects of bepridil on the electrophysiologic properties of isolated canine and rabbit myocardial fibers. Am Heart J 1986; 111: 271-9.

9. Kang L, Zheng MQ, Morishima M, Wang Y, Kaku T, Ono K. Bepridil up-regulates cardiac $\mathrm{Na}+$ channels as a long-term effect by blunting proteasome signals through inhibition of calmodulin activity. Br J Pharmacol 2009; 157: 404-14.

10. Nishida K, Fujiki A, Sakamoto T, et al. Bepridil reverses atrial electrical remodeling and L-type calcium channel down-regulation in a canine model of persistent atrial tachycardia. J Cardiovasc Electrophysiol 2007; 18: 765-72.

11. Yasuda M, Nakazato Y, Sasaki A, et al. Clinical evaluation of adverse effects during bepridil administration for atrial fibrillation and flutter. Circ J 2006; 70: 662-6.

12. Niwano S, Sasaki T, Kurokawa S, et al. Predicting the efficacy of antiarrhythmic agents for interrupting persistent atrial fibrillation according to spectral analysis of the fibrillation waves on the surface ECG. Circ J 2009; 73: 1210-8.

13. Niwano S, Wakisaka Y, Kojima J, et al. Monitoring the progression of the atrial electrical remodeling in patients with paroxysmal atrial fibrillation. Circ J 2003; 67: 133-8.

14. Yamashita T, Ogawa S, Sato T, et al. Dose-response effects of bepridil in patients with persistent atrial fibrillation monitored with transtelephonic electrocardiograms: a multicenter, randomized, placebo-controlled, double-blind study (J-BAF Study). Circ J
2009; 73: 1020-7.

15. Nobe $\mathrm{S}$, Aomine $\mathrm{M}$, Arita $\mathrm{M}$. Bepridil prolongs the action potential duration of guinea pig ventricular muscle only at rapid rates of stimulation. Gen Pharmacol 1993; 24: 1187-96.

16. Chouabe C, Drici MD, Romey G, Barhanin J, Lazdunski M. HERG and KvLQT1/IsK, the cardiac K+ channels involved in long QT syndromes, are targets for calcium channel blockers. Mol Pharmacol 1998; 54: 695-703.

17. Yoshida T, Niwano S, Inuo K, et al. Bepridil prevents paroxysmal atrial fibrillation by a class III antiarrhythmic drug effect. Pacing Clin Electrophysiol 2003; 26: 314-7.

18. Niwano S, Ortiz J, Abe H, Gonzalez X, Rudy Y, Waldo AL. Characterization of the excitable gap in a functionally determined reentrant circuit. Studies in the sterile pericarditis model of atrial flutter. Circulation 1994; 90: 1997-2014.

19. Honjo H, Yamazaki M, Kamiya K, Kodama I. Modulation of spiral wave reentry by $\mathrm{K}^{+}$channel blockade. Circ J 2007; 71: A26-31.

20. Yamazaki M, Honjo H, Nakagawa H, et al. Mechanisms of destabilization and early termination of spiral wave reentry in the ventricle by class III antiarrhythmic agent, nifekalant. Am J Physiol Heart Circ Physiol 2007; 292: H539-48.

21. Wijffels MC, Dorland R, Allessie MA. Pharmacologic cardioversion of chronic atrial fibrillation in the goat by class IA, IC, and III drugs: a comparison between hydroquinidine, cibenzoline, flecainide, and d-sotalol. J Cardiovasc Electrophysiol 1999; 10: 178-93.

22. Wijffels MC, Dorland R, Mast F, Allessie MA. Widening of the excitable gap during pharmacological cardioversion of atrial fibrillation in the goat: effects of cibenzoline, hydroquinidine, flecainide, and d-sotalol. Circulation 2000; 102: 260-7.

23. Fujiki A, Nagasawa H, Sakabe M, et al. Spectral characteristics of human atrial fibrillation waves of the right atrial free wall with respect to the duration of atrial fibrillation and effect of class I antiarrhythmic drugs. Jpn Circ J 2001; 65: 1047-51.

24. Sasaki T, Niwano S, Sasaki S, et al. Long-term follow-up of changes in fibrillation waves in patients with persistent atrial fibrillation: spectral analysis of surface ECG. Circ J 2006; 70: 169-73.

25. Fujiki A, Sakabe M, Nishida K, et al. Drug-induced changes in fibrillation cycle length and organization index can predict chemical cardioversion of long-lasting atrial fibrillation with bepridil alone or in combination with aprindine. Circ J 2004; 68: 1139-45.

26. Kurita T, Yasuda M, Nakazato Y, et al. Proarrhythmia of bepridil. Jpn J Electrocardiol 2007; 27: 61-73. (Japanese) 